\title{
Numerical studies of light-matter interaction driven by plasmonic fields: the velocity gauge
}

\author{
A. Chacón ${ }^{\mathrm{a}}$, M. Lewenstein ${ }^{\mathrm{a}, \mathrm{b}}$, M. F. Ciappina ${ }^{\mathrm{c}}$ \\ ${ }^{a}$ ICFO-Institut de Ciènces Fotòniques, Av. Carl Friedrich Gauss 3, 08860 Castelldefels \\ (Barcelona), Spain \\ ${ }^{b}$ ICREA-Institució Catalana de Recerca $i$ Estudis Avançats, Lluis Companys 23, 08010 \\ Barcelona, Spain \\ ${ }^{c}$ Max-Planck-Institut für Quantenoptik, Hans-Kopfermann-Str. 1, 85748 Garching, \\ Germany
}

\begin{abstract}
Theoretical approaches to strong field phenomena driven by plasmonic fields are based on the length gauge formulation of the laser-matter coupling. From the theoretical viewpoint it is known there exists no preferable gauge and consequently the predictions and outcomes should be independent of this choice. The use of the length gauge is mainly due to the fact that the quantity obtained from finite elements simulations of plasmonic fields is the plasmonic enhanced laser electric field rather than the laser vector potential. In this paper we develop, from first principles, the velocity gauge formulation of the problem and we apply it to the high-order harmonic generation (HHG) in atoms. A comparison to the results obtained with the length gauge is made. It is analytically and numerically demonstrated that both gauges give equivalent descriptions of the emitted HHG spectra resulting from the interaction of a spatially inhomogeneous field and the single active electron (SAE) model of the helium atom. We discuss, however, advantages and disadvantages of using different gauges in terms of numerical efficiency.

Keywords: Strong field phenomena, time dependent Schrödinger equation, plasmonic fields
\end{abstract}

\section{Introduction}

Nowadays there exists a high demand for coherent light sources extending from the ultraviolet (UV) to the extreme ultraviolet (XUV) spectral ranges. 
These sources provide important tools for basic research, material science and biology among other branches [1]. An important obstacle preventing these sources from reaching high efficiency and large duty cycles is their demanding infrastructure. The recent demonstration of XUV generation driven by surface plasmon resonances, conceived as light enhancers, could provide a plausible solution to this problem [2]. The high-order-harmonic generation (HHG) in atoms using plasmonics fields, generated starting from tailored metal nanostructures, requires no extra amplification of the incoming pulse. By exploiting the so-called surface plasmon polaritons (SPP), the local electric fields can be enhanced by several orders of magnitude [2, 3, 4], thus exceeding the threshold laser intensity for HHG generation in noble gases. One additional advantage is that the pulse repetition rate remains unaltered without any extra pumping or cavity attachment. Furthermore, the high-harmonics radiation, generated from each nanostructure typically in the UV to XUV range, acts as a source with point-like properties, enabling collimation or focusing of this coherent radiation by means of constructive interference. This opens a wide range of possibilities to spatially arrange nanostructures to enhance or shape the spectral and spatial properties of the outgoing coherent radiation in numerous ways.

One can shortly describe the high-order-harmonic generation based on plasmonics fields as follows (a more exhaustive description can be found in the seminal paper of Kim et al. [2]): a femtosecond low-intensity laser pulse is coupled to the plasmon mode of a metal nanostructure inducing a collective oscillation of the free electrons within the metal. These free charges redistribute the electric field of the laser around each of the nanostructures, thereby forming a spot of highly enhanced electric field, also known as hot spot. The plasmon amplified field exceeds the threshold of HHG, thus by injection of a gas jet, typically a noble gas, onto the spot of the enhanced field, high order harmonics from the gas atoms are generated. In the original experiment of Kim et al. [2], the output laser beam emitted from a lowpower femtosecond oscillator was directly focused onto a $10 \times 10 \mu \mathrm{m}$ size array of bow-tie nanoantennas with a pulse intensity of the order of $10^{11}$ $\mathrm{W} / \mathrm{cm}^{2}$, which is about two orders of magnitude smaller than the threshold intensity to generate HHG in noble gas atoms. The experimental result of Ref. [2] showed that the field intensity enhancement factor exceeded $20 \mathrm{~dB}$, i.e. the enhanced laser intensity is two orders of magnitude larger than the input one, which is enough to produce from the 7th to the 21st harmonics of the fundamental frequency by injecting xenon gas. For the case of 
the laser wavelength corresponding to a Ti:Sa laser, i.e. about $800 \mathrm{~nm}$, the wavelength of the emitted coherent radiation is between $38 \mathrm{~nm}$ and $114 \mathrm{~nm}$. Additionally, each bow-tie nanostructure acts as a point-like source, thus a three-dimensional (3D) arrangement of bow-ties should enable us to perform control of the properties of generated harmonics, e.g. their polarization, in various ways by exploiting interference effects. Due to the strong confinement of the plasmonic hot spots, which are of nanometer size, the laser electric field is clearly no longer spatially homogeneous in this tiny region. Since typically electron excursions are of the same order as the size of this region, important changes in the laser-matter processes occur, see. e.g. [5, 6, 7].

So far, all of the the numerical approaches to study laser-matter processes in atoms and molecules driven by plasmonic fields, in particular HHG and ATI, are based on the length gauge of the laser-coupling formulation [8, 9, 10, 11, 12, 13, 14, 15, 16, 17, 18, 19, 20, 21, 22, 23, 25. The use of the length gauge is mainly due to the fact that the quantity obtained from finite elements simulations of plasmonic fields is the plasmonic enhanced laser electric field rather than the laser vector potential. Only a couple of papers employed an extension of the Strong Field Approximation (SFA), where an approximate version of the velocity gauge was used [5, 16]. Different descriptions of light matter interaction (c.f. Ref. [28]), which include the full spatial dependence of the electromagnetic field, are closely related to the problem presented in our contribution. There are, however, distinct differences amongst the general formulation of the non-dipole problem with the one we will tackle in the present article. For instance, the next order of the non-dipole description includes both the electric quadrople and the magnetic dipole terms, which are not present in our plasmonic fields, because the typical laser intensities are far below the ones needed to consider relevant these effects.

In this article, we concentrate our effort on the formulation and numerical implementation of the velocity gauge description of light-matter interaction driven by plasmonic fields. From a pure theoretical viewpoint, it is known the velocity gauge is more appropriate and consequently our contribution will fill the missing gap, completing the whole picture in the modeling of laser-matter processes driven by plasmonic fields.

The paper is organized as follows. In Sec. II, we shall present the velocity gauge formulation of the problem and we relate it to the length gauge, clearly showing the compatibility between them. The numerical implementation is presented in Sec. III, joint with a set of examples and a discussion about how the two different algorithms, i.e. the spectra split operator and the Crank- 
Nicolson, behave as a function of the relevant parameters. Furthermore an analysis of the computational efficiency and scaling of both formulations is presented here. The paper ends with a short summary and an outlook.

\section{Theory and gauge transformation}

Quantum mechanics governs the evolution of the systems, atoms and molecules in our case, when they interact with an extental electromagnetic field. In particular, the Time Dependent Schrödinger Equation (TDSE) [33] allows us to obtain the complete time-space evolution of the particles. From a mathematical viewpoint, there are two different, but equivalent, expressions for the Hamiltonian which describes the interactions of the whole system. As a consequence the laser-matter problem can be formulated both in the so called velocity gauge (VG) or in the length gauge (LG), indistinctly. Formally, both gauges present equivalent descriptions of the quantum problem [33], and therefore the results should not change if either the VG or LG is utilized to compute the observables of interest. Here, we detail how the gauge transformation is commonly implemented in the laser-matter interaction and in particularly when a spatial inhomogeneous field interacts with an atomic or molecular target. In general, we are interested in to describe the electron dynamics of an atomic or molecular system when it interacts with an electromagnetic field. For this case the TDSE reads (atomic units are used throughout the paper otherwise stated):

$$
H \Psi(\mathbf{r}, t)=i \frac{\partial}{\partial t} \Psi(\mathbf{r}, t),
$$

where, $H$, is the Hamiltonian of the quantum system and $\Psi(\mathbf{r}, t)$ is the electron wavefunction (EWF).

Let us define the Hamiltonian, $H_{\mathrm{V}}$, in the minimum coupling or $\mathrm{VG}$ for the electromagnetic field-matter interaction as:

$$
H_{\mathrm{V}}=\frac{1}{2}[\mathbf{p}+\mathbf{A}(\mathbf{r}, t)]^{2}+V_{0}(\mathbf{r})
$$

where, $\mathbf{p}=-i \nabla$, denotes the canonical momentum operator, $\mathbf{A}(\mathbf{r}, t)$, is the vector potential of the electromagnetic field, which in this case corresponds to a spatial inhomogeneous or plasmonic field. In Eq. (2), $V_{0}(\mathbf{r}, t)$ is the electrostatic Coulomb interaction between the charged particles. The vector 
potential for the spatial inhomogeneous field typically can be represented in the following form:

$$
\begin{aligned}
\mathbf{A}(\mathbf{r}, t) & =[1+\epsilon g(\mathbf{r})] \mathbf{A}_{\mathrm{h}}(t), \\
\mathbf{A}_{\mathrm{h}}(t) & =A_{0} f(t) \sin \left(\omega_{0} t+\varphi_{\mathrm{CEP}}\right) \mathbf{e}_{z} .
\end{aligned}
$$

Here, $\mathbf{A}_{\mathrm{h}}(t)$, denotes the homogeneous or conventional vector potential, $A_{0}$ is the amplitude of the vector potential, $\omega_{0}$, is the central frequency, $\varphi_{\mathrm{CEP}}$ is the carrier-envelope-phase (CEP) and $f(t)$ is a function which defines the time envelope of the field. $\epsilon$ is a small parameter that governs the strength of the spatial inhomogeneity (see e.g. [5] for more details) and $g(\mathbf{r})$ describe the spatial dependence of the plasmonic field. Note that in the limit when $\epsilon=0$, the vector potential field does not depend on the spatial coordinate anymore and we recover the conventional laser-matter formulation. The units of $\epsilon$ depend on the function $g(\mathbf{r})$. For instance, if $g(\mathbf{r})=z$ (a linear function), $\epsilon$ has units of inverse length (see e.g. [5]).

Often, it is desirable to solve the TDSE in the length gauge or the maximal coupling gauge. This is so because the numerical or analytical calculation can be expressed in an easy way and the computation of certain observables is more efficient [29]. Therefore, the main question is how we can perform the transformation of the Hamiltonian in the VG, Eq. (2), to the LG. The gauge transformation should be boiling down in an unitary translation of the whole wavefunction [24]. We define this unitary transformation according to:

$$
\Psi_{\mathrm{V}}=Q^{\dagger} \Psi_{\mathrm{L}}
$$

where, $\Psi_{\mathrm{V}}=\Psi_{\mathrm{V}}(\mathbf{r}, t)$ and $\Psi_{\mathrm{L}}=\Psi_{\mathrm{L}}(\mathbf{r}, t)$ are the wavefunctions in the VG and LG, respectively. $Q$ is the unitary hermitian operator defined according to the following rule $Q=\exp [i \chi(\mathbf{r}, t)]\left[24,32\right.$, 33], with $\chi(\mathbf{r}, t)=\int_{\mathrm{C}}^{\mathbf{r}} \mathbf{A}\left(\mathbf{r}^{\prime}, t\right)$. $d \mathbf{r}^{\prime}$. The latter expression is a contour integral which is independent of the path, because we can safely assume that the effect of the magnetic field is negligible, i.e. that the curl of the vector potential for the inhomogeneous field is zero, $\nabla \times \mathbf{A}=\mathbf{0}$. Furthermore, by using Eqs. (1) and (4), we find the transformation for the Hamiltonian, $H_{\mathrm{V}}$, from the VG to the LG:

$$
Q H_{\mathrm{V}} Q^{\dagger} \Psi_{\mathrm{L}}=\frac{\partial \chi(\mathbf{r}, t)}{\partial t} \Psi_{\mathrm{L}}+i \frac{\partial \Psi_{\mathrm{L}}}{\partial t}
$$

Then, knowing that $\mathbf{E}(\mathbf{r}, t)=-\frac{\partial}{\partial t} \mathbf{A}(\mathbf{r}, t)$, i.e. the relationship between 
$\mathbf{E}(\mathbf{r}, t)$ and $\mathbf{A}(\mathbf{r}, \mathrm{t})$, the last expression becomes:

$$
\left[Q H_{\mathrm{V}} Q^{\dagger}+\int_{\mathrm{C}}^{\mathbf{r}} \mathbf{E}\left(\mathbf{r}^{\prime}, t\right) \cdot d \mathbf{r}^{\prime}\right] \Psi_{\mathrm{L}}=i \frac{\partial \Psi_{\mathrm{L}}}{\partial t} .
$$

As the TDSE is gauge invariant, we infer that the Hamiltonian in the VG, $H_{\mathrm{V}}$, is transformed to the $\mathrm{LG}, H_{\mathrm{L}}$, via:

$$
H_{\mathrm{L}}=Q H_{\mathrm{V}} Q^{\dagger}+\int_{\mathrm{C}}^{\mathbf{r}} \mathbf{E}\left(\mathbf{r}^{\prime}, t\right) \cdot d \mathbf{r}^{\prime}
$$

It can be demonstrated that the first term on the right hand side of Eq. (7), yields $Q H_{\mathrm{V}} Q^{\dagger}=\frac{1}{2} \mathbf{p}^{2}+V_{0}(\mathbf{r})$. Then, the Hamiltonian in the LG takes the form:

$$
H_{\mathrm{L}}=\frac{\mathbf{p}^{2}}{2}+V_{0}(\mathbf{r})+V_{\text {int }}(\mathbf{r}, t)
$$

here, $\mathbf{p}$ is the kinetic momentum operator, and $V_{\text {int }}(\mathbf{r}, t)=\int_{\mathrm{C}}^{\mathbf{r}} \mathbf{E}\left(\mathbf{r}^{\prime}, t\right) \cdot d \mathbf{r}^{\prime}$, is a contour integral. In terms of Classical Mechanics, we can interpret this last term as the work done in the electric field $\mathbf{E}\left(\mathbf{r}^{\prime}, t\right)$ to move the electron from an arbitrary place to the position $\mathbf{r}$. In the particular case when the vector potential has the functional form given by Eq. (3) and the function $g(\mathbf{r})$, is set to $g(\mathbf{r})=z$, the Hamiltonian operator in the LG becomes:

$$
H_{\mathrm{L}}=\frac{\mathbf{p}^{2}}{2}+V_{0}(\mathbf{r})+z\left(1+\frac{\epsilon}{2} z\right) E_{\mathrm{h}}(t)
$$

where, $E_{\mathrm{h}}(t)=-\frac{\partial}{\partial t} A_{\mathrm{h}}(t)$ denotes the spatial homogeneous part of the laser electric field. Commonly this field, $E_{\mathrm{h}}(t)$, is called the conventional or spatial homogeneous field.

In the next section, we shall compare the numerical accuracy of the VG and LG predictions for the high-order harmonic generation (HHG) driven by plasmonic fields. Our numerical models are based on Eqs. (2), for the VG, and (9), for the LG, respectively.

\section{Numerical algorithms}

The methods utilized to numerically integrate the TDSE are classified by considering how the time evolution of the EWF is computed. When the EWF at a later time is obtained from the one at the current time, we have 
the so-called explicit methods. On the other hand, implicit schemes find the EWF by solving an equation involving both the actual EWF and one at later time. We choose the Spectral-Split Operator (SO) method joint with the Crank-Nicolson $(\mathrm{CN})$ scheme, which are both explicit methods, to numerically integrate the TDSE of our interest. The SO uses a spectral technique to evaluate the derivative operator in the Fourier domain [31, 34], and, on the other hand, the $\mathrm{CN}$ is based on the finite element difference discretization technique 34 to implement the second derivative present in the Hamiltonian, which defines the kinetic operator term.

In order to test the accuracy of both the VG and the LG in the HHG driven by plasmonic fields, we have implemented the TDSE via the SO and CN techniques within a one spatial dimension model (1D).

A general solution of the TDSE is done by employing a unitary $U\left(t_{0}+\Delta t, t_{0}\right)$ evolution operator, where $t_{0}$ is the initial time, i.e. the initial EWF $\Psi_{0}\left(t_{0}\right)$ is known and we evolve the system to an unknown state $\Psi\left(t_{0}+\Delta t\right)$ at a given time $t_{0}+\Delta t[33]$ :

$$
\Psi\left(t_{0}+\Delta t\right)=U\left(t_{0}+\Delta t, t_{0}\right) \Psi_{0}\left(t_{0}\right)
$$

For simplicity, in Eq. (10), we have dropped out the spatial (r) dependence on the EWF. In the laser-matter community, the $U\left(t_{0}+\Delta t, t_{0}\right)$ is commonly known as a propagator and it has the following explicit form, $U\left(t_{0}+\Delta t, t_{0}\right)=$ $\exp \left[-i \int_{t_{0}}^{t_{0}+\Delta t} H\left(t^{\prime}\right) d t^{\prime}\right]$.

In reference [31, Feit et al. have introduced the SO method to numerically solve the TDSE in two spatial dimensions (2D) by using Eq. (10). This method consists in to split the time evolution operator $U\left(t_{0}+\Delta t, t_{0}\right) \approx$ $e^{-i H\left(t_{0}+\frac{\Delta t}{2}\right) \Delta t}$ in three parts [31]:

$$
\Psi\left(t_{0}+\Delta t\right)=e^{-i \frac{1}{2} \mathbf{p}^{2} \Delta t / 2} e^{-i V_{\text {eff }}\left(t_{0}+\frac{\Delta t}{2}\right) \Delta t} e^{-i \frac{1}{2} \mathbf{p}^{2} \Delta t / 2} \Psi_{0}\left(t_{0}\right) .
$$

Here, the Hamiltonian, $H\left(t_{0}+\frac{\Delta t}{2}\right)$, is divided in $H\left(t_{0}+\frac{\Delta t}{2}\right)=\frac{1}{2} \mathbf{p}^{2}+V_{\text {eff }}\left(t_{0}+\right.$ $\left.\frac{\Delta t}{2}\right)$, with $V_{\text {eff }}(t)=V_{0}(\mathbf{r})+\int_{\mathrm{C}}^{\mathbf{r}} \mathbf{E}\left(\mathbf{r}^{\prime}, t\right) \cdot d \mathbf{r}^{\prime}$ the effective potential in the LG. The main advantage of Eq. (11) is that we can evaluate the kinetic operator term, $e^{-i \frac{1}{2} \mathbf{p}^{2} \Delta t / 2} \Psi_{0}\left(\mathbf{r}, t_{0}\right)$, acting on the initial state, in the momentum space. This means that we need to compute a Forward Fourier Transform (FFT) [26] of $\Psi_{0}\left(\mathbf{r}, t_{0}\right)$ and then multiply it by a phase factor which evaluates the action of the kinetic operator, instead of a complicated derivate operator. Then, over this momentum space EWF, an Inverse Fourier Transform (IFT) is applied 
in order to return to the coordinate space [31]. This procedure is performed because the momentum operator in the conjugate (momentum) space is just a number and not a derivative one.

For the conventional or homogeneous fields case, the vector potential does not depend on the spatial coordinate, i.e. $\mathbf{A}(\mathbf{r}, t)=\mathbf{A}(t)$, allowing us to evaluate the kinetic operator in the VG as:

$$
\Psi\left(t_{0}+\Delta t\right)=e^{-i \frac{1}{2}\left(\mathbf{p}+\mathbf{A}\left(t_{0}+\Delta t / 2\right)\right)^{2} \Delta t / 2} e^{-i V_{0} \Delta t} e^{-i \frac{1}{2}\left(\mathbf{p}+\mathbf{A}\left(t_{0}+\Delta t / 2\right)\right)^{2} \Delta t / 2} \Psi_{0}\left(t_{0}\right) .
$$

Clearly, this is not the case for the spatial nonhomogeneous fields. The dependence of the vector potential on the position, as stated in Eq. (3), does not allow us to apply Eq. (12). This is so because in the momentum space the position operator becomes a derivative, which complicates substantially the SO method. Therefore, we conclude that the SO method can not be easily employed to numerically integrate the TDSE in the VG. However, by using a finite element grid discretization, we will show that the CN method can be used in both gauges, VG and LG. The CN method is based on the solution of Eq. (10) by the Caley formula and the evaluation of the kinetic operator in the position space using a finite element method [34]. In 1D the numerical algorithm can be written as:

$$
\left[1+i \frac{\Delta t}{2} H\left(t_{0}+\Delta t / 2\right)\right] \Psi\left(t_{0}+\Delta t\right)=\left[1-i \frac{\Delta t}{2} H\left(t_{0}+\Delta t / 2\right)\right] \Psi_{0}\left(t_{0}\right) .
$$

The unknown EWF, $\Psi\left(t_{0}+\Delta t\right)$, is then computed by solving a tridiagonal system of equations.

\section{System description and results}

In Attosecond Science, high-order harmonic generation (HHG) is one of the most important phenomena. For instance, it is possible to synthesize attosecond pulses or to obtain structural information about the atomic of molecular systems [1] from the HHG spectra. Therefore, we chose here this observable driven by conventional (homogeneous) and non-homogeneous fields to compare the accuracy of our VG and LG implementations.

For simplicity, we restrict ourselves to a one dimensional (1D) model, although it is known this approach is able to accurately reproduce the main features of the HHG spectra of real atoms [36]. The potential well, $V_{0}(x)$, 
which defines our atomic system, is a soft-core or quasi Coulomb potential:

$$
V_{0}(x)=-\frac{Z}{\sqrt{x^{2}+a}},
$$

where $Z$ is the atomic charge and $a$ a parameter which allows us to tune the ionization potential of the atom of interest. In this paper, we set $Z=1$ and $a=0.488$ a.u., such as the ionization potential is $I_{p}=0.9$ a.u. $(24.6 \mathrm{eV})$, i.e the value for the single active electron (SAE) model of the He atom [27]. Our ground state was computed via imaginary time propagation for a different set of spatial grid steps $\delta x$. To assure a "good time" step, $\delta t$, convergence, we have used the criterion: $\delta t<\delta x^{2} / 2$ (for more details see e.g. [34]).

In order to compute the HHG spectra, we firstly calculate the dipole acceleration expectation value, $a_{d}(t)$, as a function of time:

$$
a_{d}(t)=\left\langle\Psi(t)\left|\frac{\partial V_{0}(x)}{\partial x}+E(x, t)\right| \Psi(t)\right\rangle,
$$

where the $\mathrm{EWF} \Psi(x, t)$ is obtained via the $\mathrm{SO}$ and $\mathrm{CN}$ methods already described in the previous Section. The spectral intensity, $I_{\mathrm{HHG}}(\omega)=\left|\tilde{a}_{d}(\omega)\right|^{2}$, of the harmonic emission is then computed by Fourier transforming the dipole acceleration by using:

$$
\tilde{a}_{d}(\omega)=\int_{-\infty}^{+\infty} d t^{\prime} a_{d}\left(t^{\prime}\right) e^{i \omega t^{\prime}} .
$$

The numerical computation of the HHG spectra will be performed by using a set of position steps $\delta x=\{0.05,0.1,0.15, \ldots\}$ a.u. Consequently, and in order to estimate the numerical convergence of the HHG spectra as a function of $\delta x$, we use the spectral intensity difference between the smallest step, i.e. $\delta x_{0}=$ 0.05 a.u., and the rest of the set, $\Delta I_{\mathrm{HHG}, \delta \mathrm{x}_{\mathrm{j}}}(\omega)=\left|I_{\mathrm{HHG}, \delta \mathrm{x}_{0}}(\omega)-I_{\mathrm{HHG}, \delta \mathrm{x}_{\mathrm{j}}}(\omega)\right|$, with $j=\{1,2, \ldots\}$. Furthermore, for each of the $\delta x$, the computing time is also retrieved for both the VG and LG.

\section{1. $H H G$ driven by conventional fields}

Firstly, we present computations of the harmonic spectra intensity, $I_{\mathrm{HHG}}(\omega)$, driven by a conventional homogeneous field. This case is the limit $\epsilon \rightarrow 0$. Both numerical methods above described, i.e. the $\mathrm{SO}$ and $\mathrm{CN}$, have been used to compute the emitted harmonic spectra intensity both in the VG and LG. We shall show below that both gauges give the same results. 

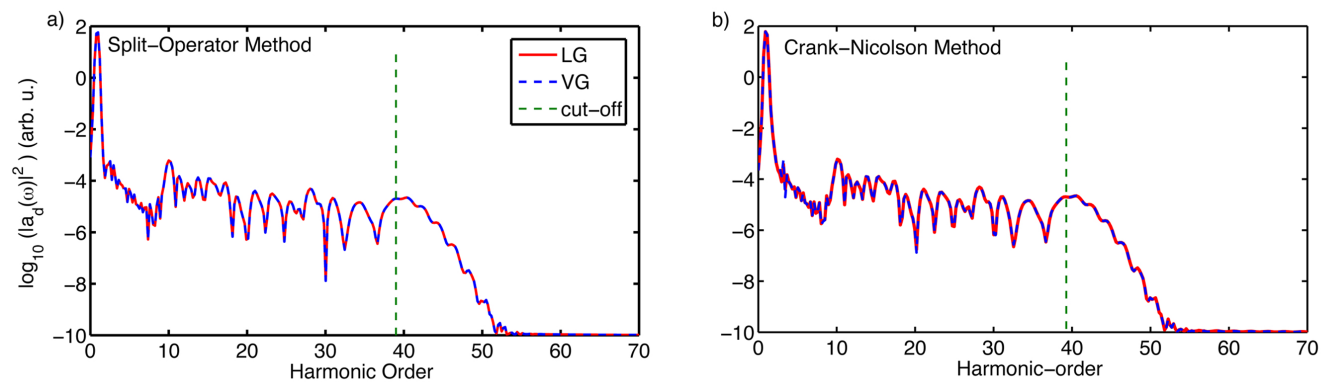

Figure 1: (color online) Computed high-order harmonic intensity spectra (in arbitrary units) driven by a spatial homogeneous (conventional) laser field under the LG (red solid line) and the VG (blue dashed line). Panel (a) the HHG spectra are obtained by using the SO method, panel (b) the same as (a) but using the CN method. The green vertical dashed line depicts the classical harmonic cut-off law, i.e., $n_{c}=\left(I_{p}+3.17 U_{p}\right) / \omega_{0}$ 35. The laser pulse parameters for these simulations are: intensity $I_{0}=2 \times 10^{14} \mathrm{~W} / \mathrm{cm}^{2}$, carrier frequency $\omega_{0}=0.057$ a.u. (corresponding to a wavelength of $\lambda=800 \mathrm{~nm}$ ), and CEP, $\varphi_{\mathrm{CEP}}=0 \mathrm{rad}$. The pulse envelope is a $\sin ^{2}$ function with four total cycles. We chose a grid step of $\delta x=0.05$ a.u. for both gauges.

The TDSE calculations are performed in a grid with a step $\delta x=0.05$ a.u., and a spatial grid length of $L_{x}=3500$ a.u. The real-time evolution is done with a time-step of $\delta t=0.00125$ a.u. Fig. 1 1 shows the spectral intensity of the harmonic emission when a laser pulse interacts with our 1D helium model. In Fig. 1(a), the comparison of the HHG spectra between the LG and VG is depicted by using the SO method. The same comparison is shown in Fig. 1(b), but here the CN method is used for the numerical integration of the TDSE. Both methods show a perfect agreement when the LG and VG are used to compute the spectral harmonic intensity. This confirms that our numerical methods are able to describe the HHG process for any of the grid steps used in our simulations.

As a next test, we have integrated the TDSE for a set of grid steps, $\delta x=$ $\{0.05,0.15, \ldots, 0.45\}$ a.u., and computed the emitted harmonics. Figure 2 , shows the results of the harmonic intensity, $I_{\mathrm{HHG}}(\omega)$, as a function of the grid step computed by the SO Figs. 2(a)-(b) and the CN Figs. 2(c)-(d) methods, respectively. For both the VG and LG, the numerical spectra by using the SO method shows a perfect agreement for all the set of grid steps, $\delta x$ used in our simulations. In contrast, the situation is different when the $\mathrm{CN}$ method is employed to compute the harmonic spectra. For instance, the Figs. 2(c)-(d) 

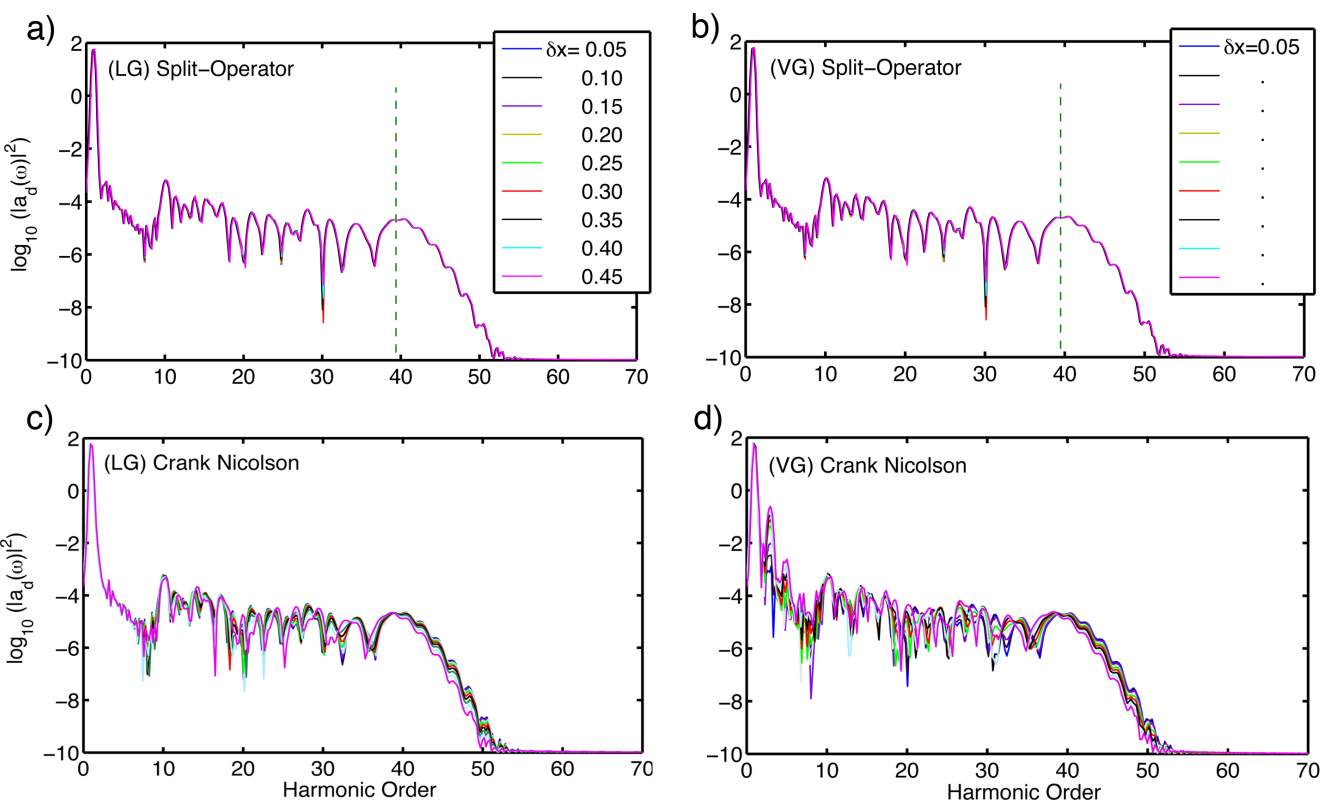

Figure 2: (color online) Computed high-order harmonic intensity spectra driven by a spatial homogeneous (conventional) field (in arbitrary units) as a function of the grid step, $\delta x$, under the LG and the VG by the SO method, panels (a)-(b) and the CN method panels (c)-(d). The green vertical dashed line depicts the classical harmonic cut-off law. The laser pulse parameters for these simulations are the same as in Fig. 1, i.e. intensity $I_{0}=2 \times 10^{14} \mathrm{~W} / \mathrm{cm}^{2}$, carrier frequency $\omega_{0}=0.057$ a.u. (corresponding to a wavelength of $\lambda=800 \mathrm{~nm}$ ), and CEP, $\varphi_{\mathrm{CEP}}=0 \mathrm{rad}$. The pulse envelope is a $\sin ^{2}$ function with four total cycles. 
show that the emitted harmonic spectrum depends on the grid step when the LG and VG are employed to computed the HHG. In addition, the computed HHG spectra slightly differ in the whole harmonic-order range whether the $\mathrm{LG}$ or the VG is used in the calculation and for the larger grid steps, i.e., $\delta x \geq$ 0.25 a.u. Additional structures can be observed in the low-order harmonics for the case of VG (see Figs. 2(d)), although the general shape, including the harmonic cutoff, is in excellent agreement with the rest of the schemes. Considering the numerical error that the finite element method has for the second derivative as a function of the grid spacing $\delta x$, it is reasonable to attribute poor convergence when the $\mathrm{CN}$ method is used with larger grid steps $\delta x$. Furthermore, in view of the fact that the VG has an extra spatial derivative of first order within the Hamiltonian, $\mathbf{p} \cdot \mathbf{A}$, we would expect that the numerical accuracy decreases when the spatial step, $\delta x$, increases. This is the reason behind the noticeable difference between the LG and the VG when larger grid steps are employed in the calculations of the HHG. Our numerical results show, however, that this difference between LG and VG disappears for the smallest spatial grid steps, i.e., $\delta x \leq 0.2$ a.u. These outcomes suggest that the best method to compute the HHG spectrum is the SO. On the other hand, in cases where the SO method is challenging, the CN method can be used if the grid step is small enough, e.g. $\delta x \lesssim 0.1$ a.u. We should note that the grid step will depend on the particular problem, i.e. laser parameters, etc., although we can expect a general trend. For this reason, we suggest to perform a convergence analysis if the $\mathrm{CN}$ method is employed and to chose the adequate parameters for the required accuracy.

In the next, we shall perform the computation of the HHG spectra driven by a spatial inhomogeneous field. For the reasons explained in Section 3, we shall only use the $\mathrm{CN}$ method and compute the harmonic emission both in the $\mathrm{LG}$ and VG.

\subsection{HHG driven by spatial inhomogeneous fields}

As was mentioned at the outset, when a laser field is focused on a metallic nanostructure, a hot spot of higher intensity, high enough to exceed the threshold for HHG in atoms, is created due to the coupling between the incoming field and the surface plasmon polaritons (SPPs) [2]. The main property of the effective laser electric field is that it presents a spatial variation in the same scale as the one of the dynamics of the active electron. Therefore, the interaction between this plamonic field and the atomic electron, which governs the HHG process, will change substantially. As the electric field is 


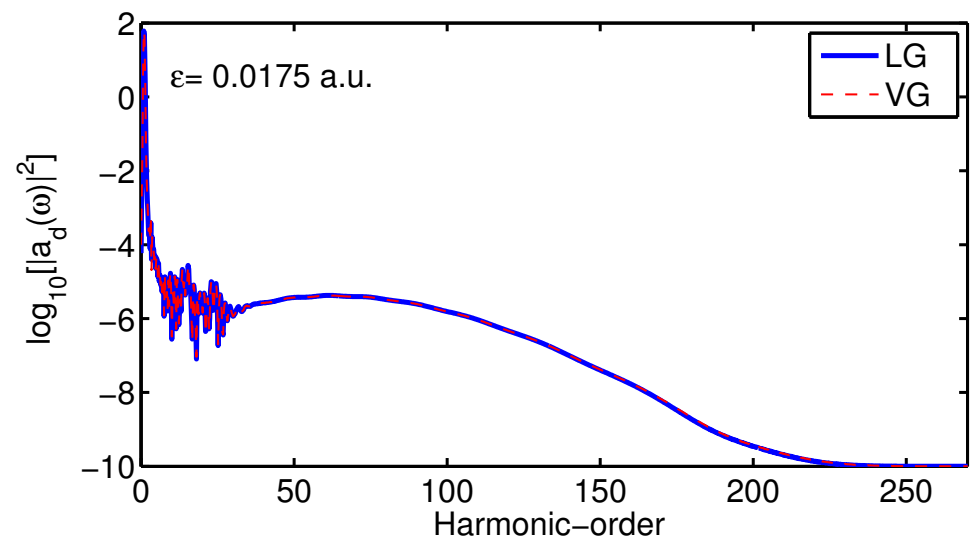

Figure 3: (color online) HHG spectra driven by a spatial inhomogeneous field computed by using the LG (blue line) and VG (red dashed line). The parameters for the laser pulse are the same that those used in Fig. 2, the inhomogeneous parameter is $\epsilon=0.0175$ a.u. (see the text for more details) and the grid step is $\delta x=0.05$ a.u.

not anymore spatially homogeneous, the electron will experience different electric field strengths along its trajectory. The question that emerges is which gauge can give us a numerical advantage when the TDSE is solved for the computation of the HHG spectra driven by spatial inhomogeneous fields. Before to address this question, we firstly demonstrate that both the LG and VG are equivalent in the description of the HHG driven by nonhomogeneous fields, as was demonstrated by the conventional case (see Section 4.1).

We have numerically integrated the TDSE in 1D for the same atomic system used in the previous section (Section 4.1), but now the effective electric field is spatially inhomogeneous. Fig. 3 shows the comparison between the calculated HHG spectra driven by an inhomogeneous field for both the LG and VG. The inhomogeneous parameter value is set at $\epsilon=0.0175$ a.u., which correspond to an inhomogeneous region of about 60 a.u. $(3 \mathrm{~nm}$ ) (see [5] for more details). Perfect agreement between the predictions of both the LG and VG are found. Therefore, these results suggest that our derivations are appropriate for spatial nonhomogeneous fields as well. As a consequence, this invariance allows us to check which gauge can be more convenient to compute the HHG driven by spatial nonhomogeneous fields. We will address this point by considering the convergence of both the LG and VG. In other words, which of the two gauges presents less numerical error against the grid 

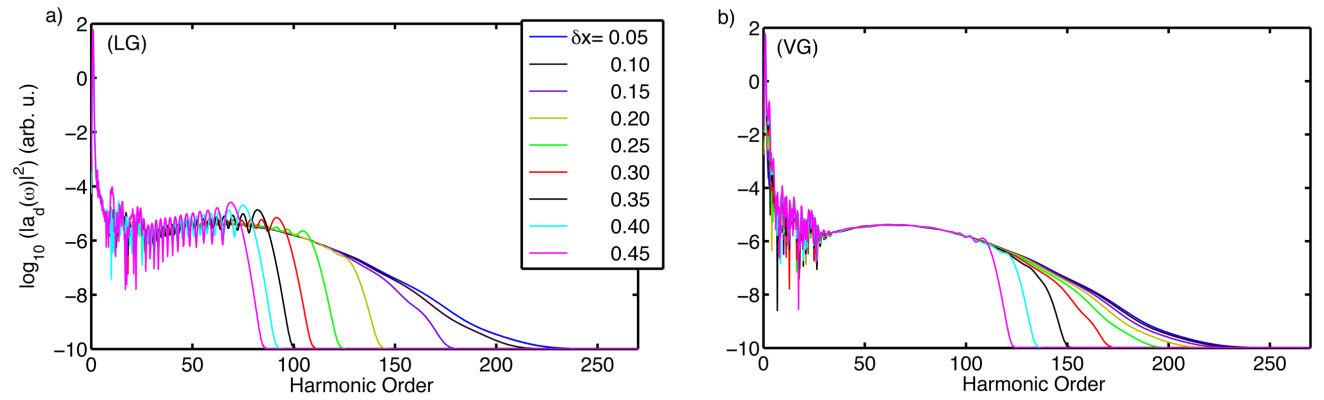

Figure 4: (color online) HHG spectra driven by plasmonic fields both in the LG and VG as a function of the grid step $\delta x$ are depicted in panel (a) and (b), respectively. We have used the CN method to numerically integrate the TDSE. The parameters for the laser pulse are the same that those used in Fig. 3 and the inhomogeneous parameter is $\epsilon=0.0175$ a.u.

step, $\delta x$, and which one is faster in the computation of the HHG spectra.

Fig. 4 shows the HHG spectra as a function of the grid step for both the LG Fig. 4(a) and the VG Fig. 4(b) computed by using the CN method. The HHG spectra for the LG show a convergence for the smallest grid step, i.e., for $\delta x=0.05$ a.u. We should note, however, that the highest frequency of the HHG spectra change when the grid step is increases, which suggests that the computation of the HHG spectra driven by spatial inhomogeneous fields deserves special attention when "large" grid steps are used. A similar result is found when the VG is employed although it is possible to observe convergence for larger values of $\delta x$. A suitable way to confirm the HHG cutoff and corroborate the convergence of the numerical schemes, is to rely on classical simulations. It is known that the limits on the HHG spectra can be obtained via classical simulations, e.g. by computing the maximum electron kinetic energy upon recombination [35]. For spatial nonhomogeneous fields, it was demonstrated a perfect agreement between the classical predictions and the TDSE simulations (see e.g. 5]) and, as a consequence, we could benchmark our VG and LG approaches by solving the classical equations of motion for an electron moving in an oscillating and spatial dependent electric field (for more details see [23]).

In addition, despite of the fact that for larger grid steps the LG shows a large deviation for the highest frequency compared to the VG results, we evaluate the relative error defined by $\frac{\Delta I_{\mathrm{HHG}, \delta \mathrm{x}_{\mathrm{j}}}}{I_{\mathrm{HHG}, \delta \mathrm{x}_{0}}}$ for each gauge as a function 

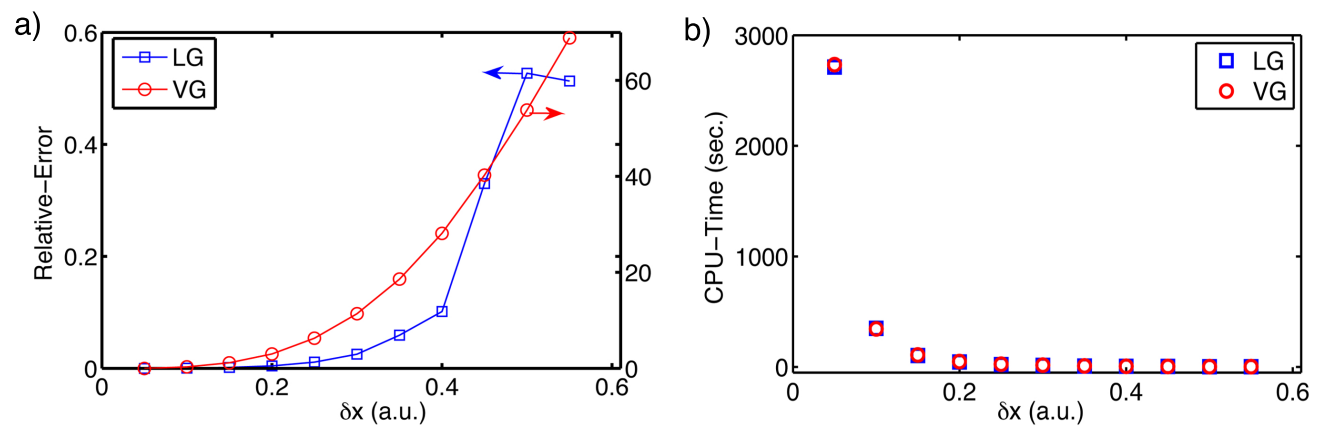

Figure 5: (color online) (a) Convergence relative-error as a function of the grid step by using the LG (blue line with squares) and the VG (red line with circles). (b) computing real-time as a function of the grid step for both the LG (blue squares) and VG (red circles). The simulation parameters are the same that those used in Fig. 4.

of the grid step $\delta x$. The results are depicted in Fig. 5(a). This panel shows that a large difference appears whether LG or VG is used to compute the HHG spectra by the CN method. For values of $\delta x$ larger than 0.2 a.u., the relative error between the LG and the VG has a difference of about two orders of magnitude, which suggests that the LG would be more appropriate than the VG to compute the HHG spectra. Finally, in the panel (b), we show the computational time for each gauge. As can be observed the computing times for both the LG and the VG are similar. From this consideration we can conclude that the LG could be the most appropriated gauge in order to compute the HHG, given the fact it allows us to use larger grid steps.

\section{Conclusions}

We have reviewed the gauge invariance problem, both analytical and numerically, for the calculation of the HHG phenomenon driven by spatial homogeneous and inhomogeneous (plasmonic enhanced) electric fields. To this purpose we have solved the TDSE in reduced dimensions by implementing the Spectral-Split Operator and the Crank-Nicolson algorithms. It was found that both the LG and VG are equivalent in the description of the harmonic emission processes for each of the two studied cases: the spatial homogeneous and the spatial inhomogeneous fields. For the spatial inhomogeneous field case, and due to the dependence of the vector potential on the position, we found that the SO method was difficult to implement in the numerical solu- 
tion of the TDSE. In contrast, the CN method has shown advantages because it is based on a finite element discretization. Our numerical results based on the CN method suggested that the calculation of the harmonic spectra depends strongly on the grid step chosen to perform the numerical integration. Both gauges are equivalent, but according to the numerical convergence of the HHG spectra, the LG apparently appears to be more accurate than the VG for the lowest harmonics. This is so because the lowest harmonics change by several orders of magnitude when the grid step increases. Furthermore, it was shown that particular attention in the choice of the spatial grid step should to be taken when spatial inhomogeneous fields are employed. This is so, because the limits of the harmonic radiation appear to be very sensitive to this parameter. Finally, it was found that the computational time was similar for both the LG or VG, if they were used for the computation of the HHG spectrum in the moderate and high laser intensity regimes.

\section{Acknowledgments}

We are grateful to Alejandro De La Calle for the insightful discussion and useful suggestions. A.C. and M.L. thanks the Spanish Ministry Project FrOntiers of QUantum Sciences (FOQUS, FIS2013-46768-P) and ERC AdG OSYRIS for financial support.

We also acknowledge the support from the ERCs Seventh Framework Programme LASERLAB-EUROPE III (grant agreement 284464) and the Ministerio de Economía y Competitividad of Spain (FURIAM project FIS201347741-R).

\section{References}

[1] F. Krausz, and M. Ivanov, Rev. Mod. Phys. 81 (2009) 163.

[2] S. Kim, J. Jin, Y.-J. Kim, I.-Y. Park, Y. Kim, and S.-W. Kim, Nature (London) 453 (2008) 757.

[3] I.-Y. Park, S. Kim, J. Choi, D.-H. Lee, Y.-J. Kim, M. F. Kling, M. I. Stockman, and S.-W. Kim, Nat. Phot. 5 (2011) 677.

[4] N. Pfullmann, C. Waltermann, M. Noack, S. Rausch, T. Nagy, M. Kovačev, V. Knittel, R. Bratschitsch, D. Akemeier, A. Hütten, A. Leitenstorfer, and U. Morgner, New J. Phys. 15 (2013) 093027. 
[5] M. F. Ciappina, J. Biegert, R.Quidant, and M. Lewenstein, Phys. Rev. A 85 (2012) 033828.

[6] M. F. Ciappina, S. S. Aćimović, T. Shaaran, J. Biegert, R. Quidant, and M. Lewenstein, Opt. Express 20 (2012) 26261.

[7] J. A. Pérez-Hernández, M. F. Ciappina, M. Lewenstein, L. Roso, and A. Zaïr, Phys. Rev. Lett. 110 (2013) 053001.

[8] A. Husakou, S.-J. Im, and J. Herrmann, Phys. Rev. A 83 (2011) 043839.

[9] I. Yavuz, E. A. Bleda, Z. Altun, and T. Topcu, Phys. Rev. A 85 (2012) 013416.

[10] T. Shaaran, M. F. Ciappina, and M. Lewenstein, Phys. Rev. A 86 (2012) 023408 .

[11] M. F. Ciappina, J. A. Pérez-Hernández, T. Shaaran, J. Biegert, R. Quidant, and M. Lewenstein, Phys. Rev. A 86 (2012) 023413.

[12] T. Shaaran, M. F. Ciappina, and M. Lewenstein, J. Mod. Opt. 59 (2012) 1634 .

[13] T. Shaaran, M. F. Ciappina, and M. Lewenstein, Ann. Phys. (Berlin) 525 (2013) 97.

[14] B. Fetić, K. Kalajdžić, and D. B.Milošević, Ann. Phys. (Berlin) 525 (2013) 107.

[15] T. Shaaran, M. F. Ciappina, R. Guichard, J. A. Pérez-Hernández, L. Roso, M. Arnold, T. Siegel, A. Zaïr, and M. Lewenstein, Phys. Rev. A 87 (2013) 041402.

[16] T. Shaaran, M. F. Ciappina, and M. Lewenstein, Phys. Rev. A 87 (2013) 053415 .

[17] I. Yavuz, Phys. Rev. A 87 (2013) 053815.

[18] M. F. Ciappina, J. A. Pérez-Hernández, T. Shaaran, L. Roso, and M. Lewenstein, Phys. Rev. A 87 (2013) 063833.

[19] J. Luo, Y. Li, Z. Wang, Q. Zhang, and P. Lu, J. Phys. B 46 (2013) 145602. 
[20] M. F. Ciappina, T. Shaaran, R. Guichard, J. A. Pérez-Hernández, L. Roso, M. Arnold, T. Siegel, A. Zaïr, and M. Lewenstein, Laser Phys. Lett. 10 (2013) 105302.

[21] M. F. Ciappina, J. A. Pérez-Hernández, T. Shaaran, M. Lewenstein, M. Krüger, and P. Hommelhoff, Phys. Rev. A 89 (2014) 013409.

[22] L. Q. Feng, M. Yuan, and T. Chu, Phys. Plasmas 20 (2013) 122307.

[23] M. F. Ciappina, J. A. Pérez-Hernández, and M. Lewenstein, Comp. Phys. Comm. 185 (2014) 398.

[24] J. R. Ackerhalt and P. W. Milonni, J. Opt. Soc. Am. B 1 (1984) 116.

[25] M. F. Ciappina, J. A. Pérez-Hernández, L. Roso, A. Zaïr, and M. Lewenstein, J. Phys. Conf. Ser. 601 (2015) 012001.

[26] M. Frigo and S. G. Johnson, FFTW http://www.fftw.org/.

[27] National Institute of Standards and Technology (NIST), Computational Chemistry Comparison and Benchmark DataBase, http://cccbdb.nist.gov/.

[28] S. Selst $\varnothing$, and M. Forre, Phys. Rev. A 76 (2007) 023427.

[29] E. Cormier, and P. Lambropoulos, J. Phys. B 29 (1996) 1667.

[30] D. Bauer, D. B. Milosevic, and W. Becker, Phys. Rev. A 72 (2005) 023415 .

[31] M. D. Feit, J. A. Fleck, and A. Steiger. J. Comp. Phys., 47 (1982) 412.

[32] C. Cohen-Tannoudji, B. Diu, and C. Laloe, Quantum Mechanics (Hermann/Wiley, Paris, 1977).

[33] J. J. Sakurai, Modern Quantum Mechanics, (Addison Wesley, 1994).

[34] W. H. Press, S. A. Teukolsky, W. T. Vetterling, and P. B. Flannery, Numerical Recipes in C: The Art of Scientic Computing, (Cambridge University Press, 2002).

[35] M. Lewenstein, P. Balcou, M. Y. Ivanov, A. L'Huillier, and P. B. Corkum, Phys. Rev. A 49 (1994) 2117. 
[36] M. Protopapas, C. H. Keitel, and P. L. Knight, Rep. Prog. Phys. 60 (1997) 389. 